\title{
Transient Elastography in Methotrexate Administered Patients
}

\author{
Fariborz Mansour-Ghanaei, ${ }^{1}$ Ali Erfani, ${ }^{1}$ Afshin Shafaghi, ${ }^{2}$ Farahnaz Joukar,, ${ }^{3,}$ Asghar Hajiabasi, ${ }^{4}$ Habib
} Zayeni, ${ }^{4}$ Abbas Darjani, ${ }^{5}$ Zahra Atrkar Roushan,, ${ }^{2, *}$ Alireza Mansour-Ghanaei, ${ }^{6}$ and Ehsan Hajipour

Jafroudi $^{7}$

${ }^{1}$ Gastrointestinal and Liver Diseases Research Center (GLDRC), Guilan University of Medical Sciences, Rasht, IR Iran

${ }^{2}$ GI Cancer Screening and Prevention Research Center (GCSPRC), Guilan University of Medical Sciences, Rasht, IR Iran

${ }^{3}$ Caspian Digestive Diseases Research Center (CDDRC), Guilan University of Medical Sciences, Rasht, IR Iran

${ }^{4}$ Guilan Rheumatology Research Center (GRRC), Rasht, IR Iran

${ }^{5}$ Skin Research Center, Guilan University of Medical Sciences, Rasht, IR Iran

${ }^{6}$ Shahid Beheshti University of Medical Sciences (SBMU), Tehran, IR Iran

${ }^{7}$ Tehran University of Medical Sciences, Tehran, IR Iran

"Corresponding author: Farahnaz Joukar, Caspian Digestive Diseases Research Center (CDDRC), Guilan University of Medical Sciences, Rasht, IR Iran. Tel: +98-3133535116, Fax: +98-1333534951, E-mail: farajov@gmail.com

"Corresponding author: Zahra Atrkar Roushan, GI Cancer Screening and Prevention Research Center (GCSPRC), Guilan University of Medical Sciences, Rasht, IR Iran. Tel: +98-3133535116, Fax: +98-1333534951, E-mail: roushan@gums.ac.ir

Received 2017 July 10; Accepted 2017 July 22.

\begin{abstract}
Background: As Methotrexate (MTX) may induce liver side effects or damage, evaluating the liver of the consumers of this drug by a noninvasive method, such as FibroScan, may be effective.

Objectives: The present study aimed at evaluating the progression of liver fibrosis and steatosis using the transient elastography (FibroScan) technique in patients, who are treated with MTX.

Methods: One hundred and one patients, who were treated with MTX because of different rheumatologic diagnosis and/or dermatologic disease, were included in the study. Liver stiffness was measured using FibroScan. Hepatic fibrosis was identified by converting the FibroScan results measured in kPa to the Metavir scale. Also, Fo-F4 and steatosis were graded.

Results: The assessment of steatosis using FibroScan showed mild, moderate, and severe stenosis in 15.8\%, 13.9\%, and 26.7\%, respectively. With regards to grading of hepatic fibrosis, $67.0 \%$ were graded as F0 to $\mathrm{F} 1,7.0 \%$ as $\mathrm{F} 2,7.0 \%$ as $\mathrm{F} 2$ to $\mathrm{F} 3,2.0 \%$ as $\mathrm{F} 3,11.0 \%$ as $\mathrm{F} 3$ to $\mathrm{F} 4$, and $6.0 \%$ as F4. A significant association was found between body mass index, waist circumferences, and fibrosis. The association between the severity of hepatic fibrosis and indices of alanine aminotransferase (ALT), gamma-glutamyl transpeptidase (GGT), and serum albumin was significant. No relationship was revealed between the severity of hepatic fibrosis and MTX dosage as well as duration of MTX use.

Conclusions: The authors found significant associations between hepatic steatosis and anthropometric parameters, level of albumin, drug dosage higher than $10 \mathrm{mg}$, and cumulative dosage of MTX. The severity of hepatic fibrosis and steatosis induced by MTX was mainly affected by the patients' obesity.
\end{abstract}

Keywords: Elastography, Methotrexate, Fibrosis, Liver Steatosis

\section{Background}

Methotrexate (MTX) is an effective therapeutic modality for treatment of a variety of inflammation-based disorders, such as rheumatoid arthritis, juvenile arthritis, lupus, psoriatic arthritis, myositis, vasculitis, and even some inflammatory gastrointestinal disorders, such as inflammatory bowel disease $(1,2)$. Regardless of its high efficacy, organic and metabolic toxicity of MTX is also considerable in the clinical setting. In this regard, the effects of MTX is dose-dependent so that long-term use of this drug with high dosages may lead to serious complications, such as bone marrow suppression, mucocutaneous reactions, pneumonitis, and hepatotoxicity $(3,4)$. Due to increased risk of hepatotoxicity following long-term use of MTX, serial monitoring of liver functional parameters is undeniable; however, liver tests function alone cannot reflect the depth of damage and therefore simultaneously applying other techniques, such as biopsy, may substantially increase diagnostic accuracy (5). Biopsy has remained as the gold standard for assessment of liver damage despite its uncomfortable side effects (6), yet it is recommended as the preferred method of use in most cases. Thus, the necessity of a rapid and non-invasive method, which has sufficient accuracy and repeatability, has become clear.

FibroScan is a relatively new medical imaging technique, which can assess liver stiffness through collagen quantification $(7,8)$. This method is an accurate non-

Copyright (c) 2017, Hepatitis Monthly. This is an open-access article distributed under the terms of the Creative Commons Attribution-NonCommercial 4.0 International License (http://creativecommons.org/licenses/by-nc/4.0/) which permits copy and redistribute the material just in noncommercial usages, provided the original work is properly cited. 
invasive technique that can be used for assessing the rate of liver fibrosis progression and thus can be widely used for following drug-induced side effects, such as MTX-induced side effects (9-11).

\section{Objectives}

However, a few studies have been published on the beneficial effects of this technique for assessing liver fibrosis induced by MTX. Hence, the present study aimed to evaluate the progression of liver fibrosis using the FibroScan technique in patients, who are treated with MTX.

\section{Methods}

\subsection{Study Setting and Periods}

One hundred and one patients, who were treated with MTX because of different rheumatologic disorder and/or dermatologic diseases, such as rheumatoid arthritis, psoriasis, or psoriatic arthritis, referred to private clinics or Razi hospital in Rasht in a time period of 18 months, were included in the study.

\subsection{Study Population and Sampling Technique}

The inclusion criteria were the use of MTX in the range of at least 2 years for any condition or the use of MTX for any reason and with a minimum cumulative dose of $2 \mathrm{~g}$ regardless of the duration of use. On admission, baseline characteristics, including demographics, type, history of the disease, dose of MTX, and duration of disease, were assessed by interviewing or physical examination.

\subsection{Data Collection}

After completing the patients' checklists, biochemical parameters, including liver enzymes, serum albumin, Apolipoprotein A1 (Apo-A1), coagulation markers, $\alpha 2$ macro, platelet count, and ferritin were all measured.

Liver stiffness measurement using transient elastography (FibroScan; Echosens, Paris, France), which measures liver stiffness (LS) contingent upon the computation of liver recurrence versatile wave inside the liver (12), was performed on the right lobe of the liver, in intercostal space with the patient lying in dorsal decubitus with the right arm in maximal abduction, as previously described. The tip of the transducer probe was covered with coupling gel and placed on the skin, between the rib bones at the level of the right lobe of the liver. The operator, assisted by a time-motion ultrasound image, located a liver portion at least $6-\mathrm{cm}$ thick and free of large vascular structures. When the target area was located, the operator pressed the probe button to start the measurements. The measurement depth was between 25 and $65 \mathrm{~mm}$. Then, validated measurements were performed on each patient. The results were expressed in kilopascals $(\mathrm{kPa})$. The median value was considered representative of the elastic modulus of the liver. Hepatic fibrosis was staged to convert Fibro scan results measured in $(\mathrm{kPa})$ to the Metavir scoring system as follows: F0 (no fibrosis), F1 (mild fibrosis without septa), F2 (moderate fibrosis with few septa), F3 (severe fibrosis with numerous septa without cirrhosis), and F4 (cirrhosis or advanced scarring of the liver) $(10,11)$. The steatosis was also graded as no steatosis ( $<11 \%$, So), mild steatosis ( $11 \%$ to $34 \%$, S1), moderate steatosis ( $34 \%$ to $67 \%$, S2), and marked to severe steatosis (> 67\%, S3) [12].

\subsection{Data Analysis}

For statistical analysis, Kolmogorov-Smirnov test was used to check the normality of data. Results were presented as mean \pm standard deviation (SD) for quantitative variables and summarized by absolute frequencies and percentages for categorical variables. Continuous variables were compared using one-way analysis of t test and/or non-parametric Mann-Whitney test whenever the data did not appear to have a normal distribution. Categorical variables were compared using Chi-square test or Fisher's exact test. For the statistical analysis, the SPSS statistical software version 16.0 for windows (SPSS Inc., Chicago, IL) was used. P values of 0.05 or less were considered statistically significant.

\subsection{Ethical Considerations}

The Medical Ethics Committee of Guilan University of Medical Sciences (GUMS) approved the study design, protocols, and informed consent procedure. All measurements were performed based on ethical guidelines of the 1975 declaration of Helsinki. Patients were enrolled after written informed consent was obtained. All patients consented to the study. This study was funded by gastrointestinal and liver diseases research center (GLDRC) of GUMS.

\section{Results}

One hundred and one consecutive patients, who were treated with MTX, between January 2013 and September 2015, were included. The mean age of the subjects was 53.5 years, ranging between 20 and 77 years and $22.7 \%$ were male. Most of the patients (87.1\%) were treated because of rheumatoid arthritis alone, 5.9\% because of psoriasis alone, and 5.9\% because of the presence of both diseases. The weight of the subjects ranged from 60 to $80 \mathrm{~kg}$ with the mean weight being $74.2 \pm 4.1 \mathrm{~kg}$. The mean body mass 
index (BMI) was $28.2 \pm 5.4 \mathrm{~kg} / \mathrm{m}^{2} ; 44.6 \%$ were overweight and $32.7 \%$ were obese. History of alcohol use was also revealed in only $3 \%$ of the cases. At the time of diagnosis, $6.9 \%$ were younger than 20 years and $51.5 \%$ were older than 40 years. According to the results of liver function test in patients treated with MTX, abnormal alanine aminotransferase (ALT), aspartate aminotransferase (AST) and alkaline phosphates (ALP) was found in $11.9 \%, 19.8 \%$, and $5.0 \%$ of patients, respectively. Also, an abnormal range of bilirubin, albumin, haptoglobin, and ApoA1 was also revealed in $3.0 \%, 5.0 \%, 52.5 \%$, and $22.8 \%$ respectively. Regarding abnormality of coagulation factors, abnormal partial thromboplastin time (PTT), prothrombin time (PT), and international normalized ratio (INR) was shown in $4.0 \%, 3.0 \%$, and $2.0 \%$ of patients. Moreover, reduced platelet count, serum hemoglobin, white blood cell count, and ferritin were found in $2.0 \%, 45.5 \%, 0.9 \%$, and $5.0 \%$, respectively. The cumulative dose of MTX ranged between lower than 2,000 $\mathrm{mg}$ in $23.8 \%, 2,000$ to $5,000 \mathrm{mg}$ in $40.6 \%$, and higher than $5,000 \mathrm{mg}$ in $35.6 \%$. Also, the duration of drug use was lower than 2 years in $5.0 \%, 2$ to 5 years in $19.8 \%$, and higher than 5 years in $75.2 \%$. About two-thirds of physicians (61.8\%) preferred to prescribe the drug with a weekly dosage of 7.5 to $10.0 \mathrm{mg}$. The assessment using FibroScan showed mild, moderate, and severe steatosis in $15.8 \%, 13.9 \%$, and $26.7 \%$ of patients, respectively. With regards to grading of hepatic fibrosis, $67.0 \%$ were graded as Fo to $\mathrm{F} 1,7.0 \%$ as $\mathrm{F} 2,7.0 \%$ as $\mathrm{F} 2$ to $\mathrm{F} 3,2.0 \%$ as $\mathrm{F} 3,11.0 \%$ as $\mathrm{F} 3$ to $\mathrm{F} 4$, and $6.0 \%$ as F4. With respect to the association between hepatic fibrosis and demographic characteristics (Table 1), there was no relationship between the presence of fibrosis and patients' age, age at diagnosis, the presence of underlying disease, height, and BMI, while the presence of fibrosis was more commonly found in patients with a body mass index higher than $30 \mathrm{~kg} / \mathrm{m}^{2}(\mathrm{P}=0.01)$ and also in those with waist circumference $>120 \mathrm{~cm}(\mathrm{P}=0.05)$. Also, as shown in Table 1 , the type of rheumatologic disease was not associated with the presence or absence of hepatic fibrosis.

Regarding the association between hepatic fibrosis and chemical biomarkers, abnormal levels of ALT, gammaglutamyl transpeptidase (GGT), and serum albumin was more frequent in those with hepatic fibrosis. However, no relationship was revealed between the groups with and without hepatic fibrosis, in terms of cumulative dosage of MTX and duration of drug use, as well as initial and weekly dosages of the drug (Table 2). More interestingly, no association was found between the presence and absence of hepatic fibrosis and grade of steatosis, where mild, moderate, and severe steatosis was found in $18.2 \%, 18.2 \%$, and $33.3 \%$ of cases in the group with fibrosis and $14.7 \%, 11.8 \%$, and $23.5 \%$ of cases in the group without fibrosis. Regarding the association between the grade of steatosis and base- line variables, there was no association between the grade of steatosis and patients age and height, whereas severe steatosis was significantly more prevalent in patients with body weight higher than $100 \mathrm{~kg}(\mathrm{P}=0.001)$, in patients with $\mathrm{BMI}>30 \mathrm{~kg} / \mathrm{m}^{2}(\mathrm{P}=0.007)$, and also in those with waist circumference $>120 \mathrm{~cm}$ (Table 3 ). There was no relationship between grade of steatosis and type of rheumatologic disease. Among laboratory indices, only the prevalence of abnormal albumin level was associated with the grade of steatosis. As indicated in Table 4, administrating higher cumulative dosages of MTX (> 5,000 mg) was associated with higher grades of steatosis $(P=0.03)$. As shown in Figure 1 , no association was found between the grade of steatosis and severity of hepatic fibrosis.

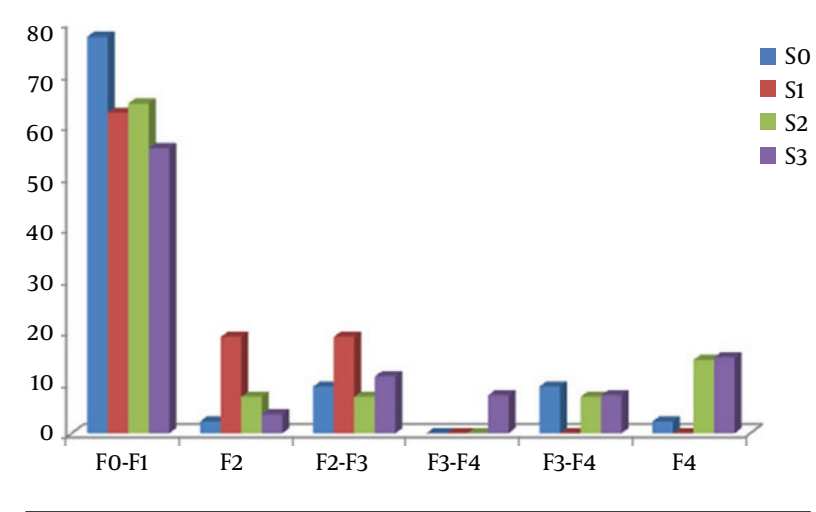

Figure 1. Association Between Severity of Fibrosis and Steatosis Grading

The results of the biopsy in patients were graded based on the Roenigk scoring system, so 3 were graded as I, 1 was graded as II, and 3 were graded as III. Among those biopsied patients, steatosis was found in all patients with grade II or III, while only in one patient with grade I. Also, hepatic fibrosis was revealed in all patients with grade II or III, while it was not found in any of the patients with grade I (Table 5).

\section{Discussion}

In the present study and by applying FibroScan, the presence and severity of hepatic fibrosis were assessed in patients, who were treated with MTX, and no significant association was shown between the grade of steatosis and severity of hepatic fibrosis. For the first time and in a prospective study in 2006, the effectiveness of FibroScan as a noninvasive diagnostic method for assessing hepatic fibrosis was assessed in patients with hepatitis $\mathrm{C}$ or HIV infection (13). They showed that compared with other noninvasive methods, FibroScan had reliable and acceptable results (14-16). Following an initial assessment, a study in 
Table 1. The Association Between Hepatic Fibrosis and Demographic Characteristics ${ }^{\mathrm{a}}$

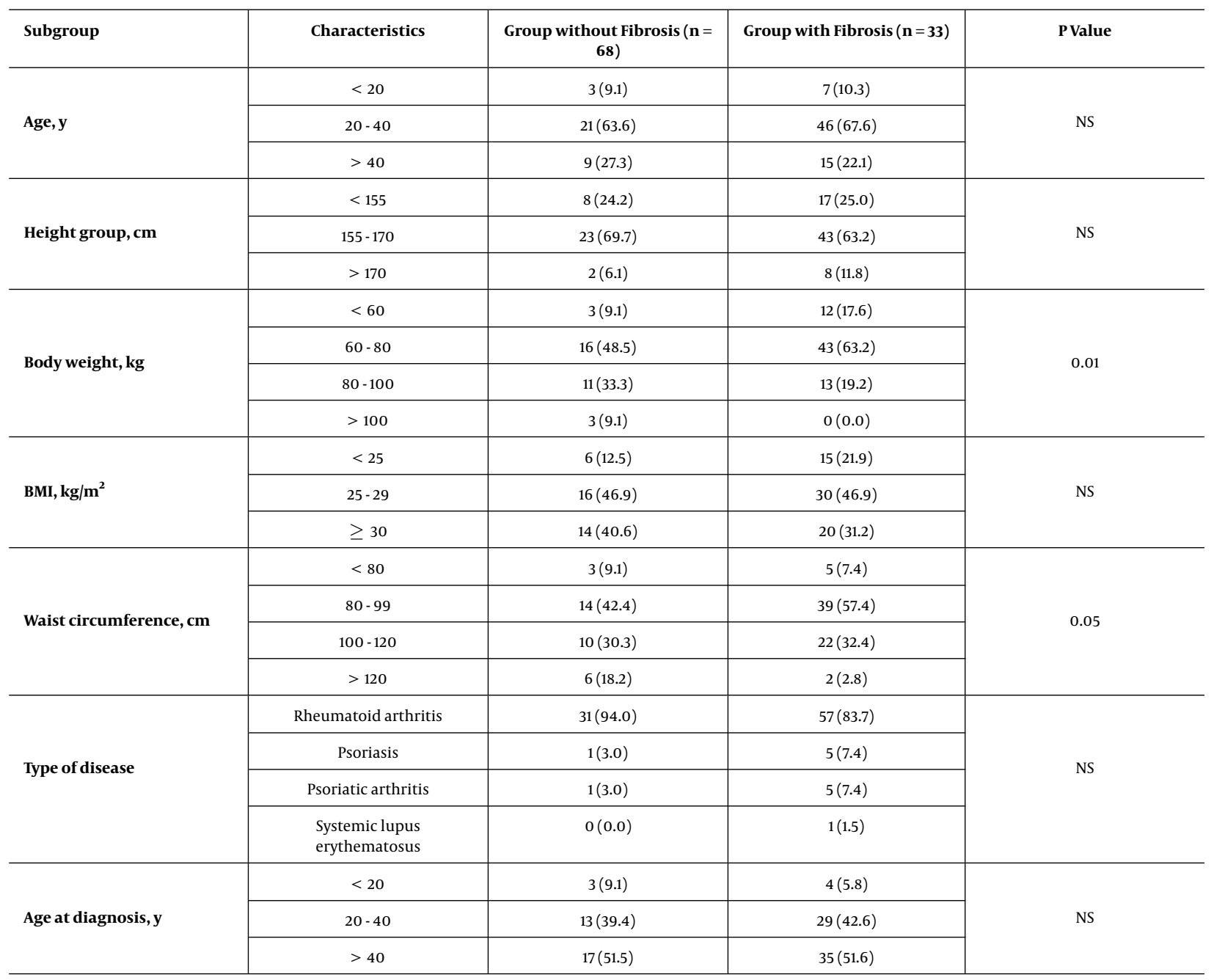

${ }^{\mathrm{a}}$ Values are expressed as No. (\%).

2006 showed rare hepatic fibrotic involvement in patients with Crohn's disease that were treated with high-dose MTX and it was finally concluded that Fibro Scan technique can be used for ruling out hepatic fibrosis in these patients (17). In this regard, it has been recommended that Fibro Scan should be used only in patients with positive Fibro Scan, especially in the presence of abnormal liver function test. As another finding, among baseline variables, only body weight and waist circumference were associated with hepatic fibrosis. In parallel with the current study, in a study by Laharie et al. (7) in 2006, it was shown that among patients with hepatic fibrosis, who were treated with methotrexate because of different inflammatory disorders, only $8.5 \%$ had severe hepatic fibrosis. Among baseline characteristics in their study, obesity and alcohol use affected the ap- pearance of hepatic fibrosis, which was consistent with the current findings. It seems that among patients' characteristics, the use of MTX in combination with obesity could increase the risk of hepatic fibrosis and steatosis. The researchers also showed no association between the presence of hepatic fibrosis and steatosis and other variables, including gender, age, age at diagnosis, as well as the type of disease that was similar to a recent study on patients with IBD treated with MTX. Also, the current study could not demonstrate the effect of drug cumulative dosages and also the duration of drug use on severity of hepatic fibrosis, which was similar to the study of Laharie et al. (7). This finding was also confirmed by other similar studies, however, contrary to the current study, Menter et al. (18) and Curtis et al. (19) showed a direct association between cumulative 
Table 2. The Association Between Hepatic Fibrosis and How to Use Drug ${ }^{\mathrm{a}}$

\begin{tabular}{|c|c|c|c|c|}
\hline & Characteristics & Group without Fibrosis $(n=68)$ & Group with Fibrosis $(n=33)$ & P Value \\
\hline \multirow{3}{*}{ Cumulative dosage, mg } & $<2000$ & $16(23.5)$ & $8(24.3)$ & \multirow{3}{*}{ NS } \\
\hline & $2000-5000$ & $27(39.7)$ & $14(42.4)$ & \\
\hline & $>5000$ & $25(36.8)$ & $11(33.3)$ & \\
\hline \multirow{3}{*}{ Duration of use, $y$} & $<2$ & $5(7.4)$ & $0(0.0)$ & \multirow{3}{*}{ NS } \\
\hline & $2-5$ & $14(20.5)$ & $6(18.2)$ & \\
\hline & $>5$ & $49(72.1)$ & $27(81.8)$ & \\
\hline \multirow{8}{*}{ Used dosage, mg } & 2.5 & $4(5.9)$ & $3(9.1)$ & \multirow{8}{*}{ NS } \\
\hline & 5 & $3(4.4)$ & $2(6.1)$ & \\
\hline & 7.5 & $19(27.9)$ & $5(15.1)$ & \\
\hline & 10 & $26(38.3)$ & $12(36.3)$ & \\
\hline & 12.5 & $1(1.5)$ & $3(9.1)$ & \\
\hline & 15 & $12(17.6)$ & $6(18.2)$ & \\
\hline & 17.5 & $1(1.5)$ & $0(0.0)$ & \\
\hline & 20 & $2(2.9)$ & $2(6.1)$ & \\
\hline \multirow{9}{*}{ Initial dosage, $\mathrm{mg}$} & 0.65 & $0(0.0)$ & $1(3.0)$ & \multirow{9}{*}{ NS } \\
\hline & 2.5 & $5(7.4)$ & $3(9.1)$ & \\
\hline & 5 & $6(8.8)$ & $2(6.1)$ & \\
\hline & 7.5 & $26(38.3)$ & $7(21.2)$ & \\
\hline & 10 & $17(25.0)$ & $11(33.3)$ & \\
\hline & 12.5 & $1(1.5)$ & $0(0.0)$ & \\
\hline & 15 & $9(13.2)$ & $7(21.2)$ & \\
\hline & 20 & $2(2.9)$ & $2(6.1)$ & \\
\hline & 22.5 & $2(2.9)$ & $0(0.0)$ & \\
\hline
\end{tabular}

${ }^{\mathrm{a}}$ Values are expressed as No. (\%)

dose of MTX and severity of hepatotoxicity.

For determining the association between the level of hepatic fibrosis and abnormality of laboratory biomarkers, the authors also measured the serum biomarkers, simultaneously. According to the current findings, among all study biomarkers, abnormality in ALT, GGT, and serum albumin was related to fibrosis severity. In other similar studies, low serum albumin level was associated with fibrosis severity and thus it was recommended to use liver biopsy in patients that received MTX with serum albumin less than $3 \mathrm{mg} / \mathrm{dL}$ (20). In conclusion, it seems that the occurrence of liver fibrosis following the use of MTX could significantly alter liver functional indices, including liver enzymes. In a similar study on cancer patients, the use of MTX led to an increase in ALT in $14 \%$ and AST in $8 \%$ of patients, although by discontinuing drug use within 1 month, the level of liver enzymes could return to the normal range (21). In the present study, no association was revealed between the grade of steatosis and severity of hepatic fibrosis that was also previously indicated in some studies (22-26). Overall, it could be concluded that FibroScan is a useful technique for assessing hepatic fibrosis in patients, who receive MTX. According to the current observation, the severity of both hepatic fibrosis and steatosis could be influenced by obesity, yet may not be affected by other demographic characteristics as well as by the dose and duration of MTX use. In contrary to the initial expectation, the severity of hepatic fibrosis was not related to the grade of steatosis assessed by FibroScan. In this study, the duration of MTX use was $<2->5$. As, continuing the drug for more than 5 years may cause more liver damage, it is recommended to study this group separately in the future.

$$
\text { Implication for health policy mak- }
$$
ers/practice/research/medical education:

Transient elastography in combination with liver function tests could be useful tools for assessment of MTX hep- 
Table 3. The Association Between Grading of Steatosis and Demographic Characteristics ${ }^{\mathrm{a}}$

\begin{tabular}{|c|c|c|c|c|c|c|}
\hline Subgroup & Characteristics & So $(n=44)$ & $S 1(n=16)$ & $S 2(n=14)$ & S3 $(\mathbf{n}=\mathbf{2 7})$ & P Value \\
\hline \multirow{3}{*}{ Age, y } & $<20$ & $6(13.6)$ & $1(6.2)$ & $0(0.0)$ & $3(11.1)$ & \multirow{3}{*}{ NS } \\
\hline & $20-40$ & $27(61.4)$ & $8(50.0)$ & $11(78.6)$ & $21(77.8)$ & \\
\hline & $>40$ & $11(25.0)$ & $7(43.8)$ & $3(21.4)$ & $3(11.1)$ & \\
\hline \multirow{3}{*}{ Height, cm } & $<155$ & $11(25.0)$ & $6(37.5)$ & $4(28.6)$ & $4(14.8)$ & \multirow{3}{*}{ NS } \\
\hline & $155-170$ & $29(65.9)$ & $8(50.0)$ & $9(64.3)$ & $20(74.1)$ & \\
\hline & $>170$ & $4(9.1)$ & $2(12.5)$ & $1(7.1)$ & $3(11.1)$ & \\
\hline \multirow{4}{*}{ Body weight, kg } & $<60$ & $13(29.5)$ & $0(0.0)$ & $0(0.0)$ & $2(7.4)$ & \multirow{4}{*}{0.001} \\
\hline & $60-80$ & $28(63.6)$ & $13(81.2)$ & $7(50.0)$ & $11(40.7)$ & \\
\hline & $80-100$ & $3(6.9)$ & $3(18.8)$ & $7(50.0)$ & $11(40.7)$ & \\
\hline & $>100$ & $0(0.0)$ & $0(0.0)$ & $0(0.0)$ & $33(11.2)$ & \\
\hline \multirow{3}{*}{ BMI, $\mathrm{kg} / \mathrm{m}^{2}$} & $<25$ & $14(30.9)$ & $2(12.5)$ & $0(0.0)$ & $5(18.5)$ & \multirow{3}{*}{0.007} \\
\hline & $25-29$ & $23(52.3)$ & $8(50.0)$ & $8(57.1)$ & $7(25.9)$ & \\
\hline & $\geq 30$ & $7(15.8)$ & $6(37.5)$ & $6(42.9)$ & $15(55.6)$ & \\
\hline \multirow{4}{*}{ Waist circumference, $\mathrm{cm}$} & $<80$ & $8(18.1)$ & $0(0.0)$ & $0(0.0)$ & $0(0.0)$ & \multirow{4}{*}{0.001} \\
\hline & $80-100$ & $31(70.5)$ & $8(50.0)$ & $4(28.6)$ & $10(37.0)$ & \\
\hline & $101-120$ & $5(11.4)$ & $8(50.0)$ & $9(64.3)$ & $10(37.0)$ & \\
\hline & $>120$ & $0(0.0)$ & $0(0.0)$ & $1(7.1)$ & $7(26.0)$ & \\
\hline \multirow{4}{*}{ Type of disease } & Rheumatoid arthritis & $40(90.9)$ & $15(93.8)$ & $11(78.6)$ & $22(81.5)$ & \multirow{4}{*}{ NS } \\
\hline & Psoriasis & $3(6.8)$ & $0(0.0)$ & $1(7.1)$ & $2(7.4)$ & \\
\hline & Psoriatic arthritis & $0(0.0)$ & $1(6.2)$ & $2(14.3)$ & $3(11.1)$ & \\
\hline & Systemic lupus erythematosus & $1(2.3)$ & $0(0.0)$ & $0(0.0)$ & $0(0.0)$ & \\
\hline \multirow{3}{*}{ Age at diagnosis, y } & $<20$ & $5(11.4)$ & $1(6.2)$ & $0(0.0)$ & $1(3.7)$ & \multirow{3}{*}{ NS } \\
\hline & $20-40$ & $22(50.0)$ & $2(12.5)$ & $7(50.0)$ & $11(40.7)$ & \\
\hline & $>40$ & $17(38.6)$ & $13(81.3)$ & $7(50.0)$ & $15(55.6)$ & \\
\hline
\end{tabular}

${ }^{\mathrm{a}}$ Values are expressed as No. (\%).

atotoxicity

The MTX may cause more significant steatosis and fibrosis among the most obese patients (BMI $>30$ and waist circumference $>120 \mathrm{~cm}$ ).

MTX-induced fibrosis may be unrelated to steatosis.

The severity of MTX-induced hepatic fibrosis is not necessarily related to the duration of treatment and drug cumulative dose.

\section{Acknowledgments}

The authors would like to thank all members of gastrointestinal and liver diseases research center (GLDRC). Also, they would like to thank all the hospital staff that assisted with this study.

\section{Footnotes}

Authors' Contribution: Fariborz Mansour-Ghanaei, Farahnaz Joukar, Asghar Hajiabasi: study concept; Fariborz Mansour-Ghanaei, Farahnaz Joukar: study design; Fariborz Mansour-Ghanaei, Farahnaz Joukar, Asghar Hajiabasi: literature search; Ali Erfani, Farahnaz Joukar, Habib Zayeni, Abbas Darjani, Alireza Mansour-Ghanaei, Ehsan Hajipour Jafroudi: data acquisition; Ali Erfani, Habib Zayeni, Abbas Darjani: data analysis/interpretation; Zahra Atrkar Roushan: statistical analysis; Zahra Atrkar Roushan Afshin Shafaghi. manuscript preparation; Ali Erfani, Fariborz Mansour-Ghanaei, Farahnaz Joukar: manuscript definition of intellectual content; Farahnaz Joukar, Fariborz Mansour-Ghanaei: manuscript editing and revision. All authors read and approved the final manuscript.

Financial Disclosure: The authors declare that they had 
Table 4. The Association Between Grading of Steatosis and How to Use the Drug ${ }^{a}$

\begin{tabular}{|c|c|c|c|c|c|c|}
\hline Subgroups & Characteristics & So $(n=44)$ & $S 1(n=16)$ & $S 2(n=14)$ & S3 $(n=27)$ & P Value \\
\hline \multirow{3}{*}{ Cumulative dosage, mg } & $<2000$ & $9(20.5)$ & $6(37.5)$ & $1(7.1)$ & $8(29.6)$ & \multirow{3}{*}{0.03} \\
\hline & $2000-5000$ & $19(43.2)$ & $9(56.2)$ & $6(42.9)$ & $7(25.9)$ & \\
\hline & $>5000$ & $16(36.3)$ & $1(6.3)$ & $7(50.0)$ & $12(44.5)$ & \\
\hline \multirow{3}{*}{ Duration of use, $y$} & $<2$ & $2(4.5)$ & $1(6.3)$ & $0(0.0)$ & $2(7.4)$ & \multirow{3}{*}{ NS } \\
\hline & $2-5$ & $8(18.2)$ & $5(31.2)$ & $2(14.3)$ & $5(18.5)$ & \\
\hline & $>5$ & $34(77.3)$ & $10(62.5)$ & $12(85.7)$ & $20(74.1)$ & \\
\hline \multirow{8}{*}{ Used dosage, mg } & 2.5 & $4(9.1)$ & $2(12.5)$ & $0(0.0)$ & $1(3.7)$ & \multirow{8}{*}{ NS } \\
\hline & 5 & $0(0.0)$ & $2(12.5)$ & $1(7.1)$ & $2(7.4)$ & \\
\hline & 7.5 & $12(27.3)$ & $3(18.8)$ & $4(28.6)$ & $5(18.5)$ & \\
\hline & 10 & $19(43.2)$ & $5(31.2)$ & $4(28.6)$ & $10(37.1)$ & \\
\hline & 12.5 & $2(4.5)$ & $0(0.0)$ & $1(7.1)$ & $1(3.7)$ & \\
\hline & 15 & $6(13.5)$ & $4(25.0)$ & $3(21.5)$ & $5(18.5)$ & \\
\hline & 17.5 & $0(0.0)$ & $0(0.0)$ & $0(0.0)$ & $1(3.7)$ & \\
\hline & 20 & $1(2.3)$ & $0(0.0)$ & $1(7.1)$ & $2(7.4)$ & \\
\hline \multirow{9}{*}{ Initial dosage, $\mathrm{mg}$} & 0.65 & $1(2.3)$ & $0(0.0)$ & $0(0.0)$ & $0(0.0)$ & \multirow{9}{*}{ NS } \\
\hline & 2.5 & $3(6.8)$ & $2(12.5)$ & $0(0.0)$ & $3(11.1)$ & \\
\hline & 5 & $1(2.3)$ & $5(31.2)$ & $1(7.1)$ & $1(3.7)$ & \\
\hline & 7.5 & $22(50.1)$ & $3(18.8)$ & $2(14.4)$ & $6(22.3)$ & \\
\hline & 10 & $10(22.6)$ & $5(31.2)$ & $3(21.4)$ & $10(37.0)$ & \\
\hline & 12.5 & $0(0.0)$ & $0(0.0)$ & $0(0.0)$ & $1(3.7)$ & \\
\hline & 15 & $5(11.3)$ & $(3 / 6) 1$ & $(9 / 42) 6$ & $4(14.8)$ & \\
\hline & 20 & $1(2.3)$ & $0(0.0)$ & $1(7.1)$ & $2(7.4)$ & \\
\hline & 22.5 & $1(2.3)$ & $0(0.0)$ & $1(7.1)$ & $0(0.0)$ & \\
\hline
\end{tabular}

${ }^{\mathrm{a}}$ Values are expressed as No. (\%).

Table 5. Finding of Fibrosis and Steatosis in Biopsy and Scan According to the Roenigk Grading System

\begin{tabular}{lccccc}
\hline Patient & Roenigk Grading & Steatosis in Biopsy & Steatosis in Scan & Fibrosis in Biopsy & Fibrosis Grade in Scan \\
\hline $\mathbf{1}$ & Grade III & + & S3 & + \\
$\mathbf{2}$ & Grade III & + & So & + \\
$\mathbf{3}$ & Grade III & + & S1 & + \\
$\mathbf{4}$ & Grade II & + & S3 & + \\
$\mathbf{5}$ & Grade I & + & S2 4 & + \\
$\mathbf{6}$ & Grade I & - & So & F3-F4 \\
$\mathbf{7}$ & Grade I & - & F4 & F2-F3 \\
\hline
\end{tabular}

no conflicts of interest concerning this article.

Funding/Support: This research received no specific grants from any funding agency in the public, commercial, or not-for-profit sectors.

\section{References}

1. Busard C, Zweegers J, Limpens J, Langendam M, Spuls PI. Combined use of systemic agents for psoriasis: a systematic review. JAMA Dermatol. 2014;150(11):1213-20. doi: 10.1001/jamadermatol.2014.1111. [PubMed: 25188393]. 
2. Feldman M, Friedman LS, Brandt LJ. Sleisenger and Fordtran's Gastrointestinal and Liver Disease E-Book: Pathophysiology, Diagnosis, Management, Expert Consult Premium Edition-Enhanced Online Features. 1. Elsevier Health Sciences; 2010.

3. Barrera P, Laan RF, van Riel PL, Dekhuijzen PN, Boerbooms AM, van de Putte LB. Methotrexate-related pulmonary complications in rheumatoid arthritis. Ann Rheum Dis. 1994;53(7):434-9. [PubMed: 7944614].

4. Lindsay K, Fraser AD, Layton A, Goodfield M, Gruss H, Gough A Liver fibrosis in patients with psoriasis and psoriatic arthritis on long-term, high cumulative dose methotrexate therapy. Rheumatology (Oxford). 2009;48(5):569-72. doi: 10.1093/rheumatology/kep023. [PubMed: 19273538].

5. Dawwas MF, Aithal GP. The quest for an evidence-based approach to surveillance for methotrexate-related hepatotoxicity: promise and perils. Br J Dermatol. 2015;172(6):1684-5. doi: 10.1111/bjd.13620. [PubMed: 25523838].

6. Valentino PL, Church PC, Shah PS, Beyene J, Griffiths AM, Feldman BM, et al. Hepatotoxicity caused by methotrexate therapy in children with inflammatory bowel disease: a systematic review and meta-analysis. Inflamm Bowel Dis. 2014;20(1):47-59. doi: 10.1097/01.MIB.0000436953.88522.3e. [PubMed: 24280876].

7. Laharie D, Zerbib F, Adhoute X, Boue-Lahorgue X, Foucher J, Castera L, et al. Diagnosis of liver fibrosis by transient elastography (FibroScan) and non-invasive methods in Crohn's disease patients treated with methotrexate. Aliment Pharmacol Ther. 2006;23(11):16218. doi:10.1111/j.1365-2036.2006.02929.x. [PubMed: 16696812].

8. Pu K, Shi JH, Wang X, Tang Q, Wang XJ, Tang KL, et al. Diagnostic accuracy of transient elastography (FibroScan) in detection of esophageal varices in patients with cirrhosis: A meta-analysis. World $J$ Gastroenterol. 2017;23(2):345-56. doi:10.3748/wjg.v23.i2.345. [PubMed: 28127208].

9. Foucher J, Chanteloup E, Vergniol J, Castera L, Le Bail B, Adhoute $\mathrm{X}$, et al. Diagnosis of cirrhosis by transient elastography (FibroScan): a prospective study. Gut. 2006;55(3):403-8. doi: 10.1136/gut.2005.069153. [PubMed: 16020491].

10. Maybury CM, Samarasekera E, Douiri A, Barker JN, Smith CH. Diagnostic accuracy of noninvasive markers of liver fibrosis in patients with psoriasis taking methotrexate: a systematic review and meta-analysis. Br J Dermatol. 2014;170(6):1237-47. doi: 10.1111/bjd.12905. [PubMed: 24588075].

11. Huang R, Jiang N, Yang R, Geng X, Lin J, Xu G, et al. Fibroscan improves the diagnosis sensitivity of liver fibrosis in patients with chronic hepatitis B. Exp Ther Med. 2016;11(5):1673-7. doi: 10.3892/etm.2016.3135. [PubMed: 27168788].

12. Sandrin L, Fourquet B, Hasquenoph JM, Yon S, Fournier C, Mal F, et al. Transient elastography: a new noninvasive method for assessment of hepatic fibrosis. Ultrasound Med Biol. 2003;29(12):1705-13. [PubMed: 14698338].

13. Castera L, Vergniol J, Foucher J, Le Bail B, Chanteloup E, Haaser M, et al. Prospective comparison of transient elastography, Fibrotest, APRI, and liver biopsy for the assessment of fibrosis in chronic hepatitis C. Gastroenterology. 2005;128(2):343-50. [PubMed: 15685546].

14. Merat S, Rezvan H, Nouraie M, Jamali A, Assari S, Abolghasemi H, et al. The prevalence of hepatitis B surface antigen and anti-hepatitis B core antibody in Iran: a population-based study. Arch Iran Med.
2009;12(3):225-31. [PubMed: 19400598].

15. Sandrin L, Tanter M, Gennisson JL, Catheline S, Fink M. Shear elasticity probe for soft tissues with 1-D transient elastography. IEEE Trans Ultrason Ferroelectr Freq Control. 2002;49(4):436-46. [PubMed: 11989699].

16. Ziol M, Handra-Luca A, Kettaneh A, Christidis C, Mal F, Kazemi F, et al. Noninvasive assessment of liver fibrosis by measurement of stiffness in patients with chronic hepatitis C. Hepatology. 2005;41(1):48-54. doi: 10.1002/hep.20506. [PubMed: 15690481].

17. American Academy of Dermatology Work G, Menter A, Korman NJ, Elmets CA, Feldman SR, Gelfand JM, et al. Guidelines of care for the management of psoriasis and psoriatic arthritis: section 6 . Guidelines of care for the treatment of psoriasis and psoriatic arthritis: case-based presentations and evidence-based conclusions. J Am Acad Dermatol. 2011;65(1):137-74. doi:10.1016/j.jaad.2010.11.055. [PubMed: 21306785].

18. Curtis JR, Beukelman T, Onofrei A, Cassell S, Greenberg JD, Kavanaugh $A$, et al. Elevated liver enzyme tests among patients with rheumatoid arthritis or psoriatic arthritis treated with methotrexate and/or leflunomide. Ann Rheum Dis. 2010;69(1):43-7. doi: 10.1136/ard.2008.101378. [PubMed: 19147616].

19. d'Assignies G, Fontes G, Kauffmann C, Latour M, Gaboury L, Boulanger Y, et al. Early detection of liver steatosis by magnetic resonance imaging in rats infused with glucose and intralipid solutions and correlation to insulin levels. Metabolism. 2013;62(12):1850-7. doi: 10.1016/j.metabol.2013.08.003. [PubMed: 24035445].

20. Berkowitz RS, Goldstein DP, Bernstein MR. Ten years' experience with methotrexate and folinic acid as primary therapy for gestational trophoblastic disease. Gynecol Oncol. 1986;23(1):111-8. doi: 10.1016/00908258(86)90123-x.

21. Browning JD, Szczepaniak LS, Dobbins R, Nuremberg P, Horton JD, Cohen JC, et al. Prevalence of hepatic steatosis in an urban population in the United States: impact of ethnicity. Hepatology. 2004;40(6):138795. doi: 10.1002/hep.20466. [PubMed: 15565570].

22. Friedrich-Rust M, Romen D, Vermehren J, Kriener S, Sadet D, Herrmann E, et al. Acoustic radiation force impulse-imaging and transient elastography for non-invasive assessment of liver fibrosis and steatosis in NAFLD. Eur J Radiol. 2012;81(3):e325-31. doi: 10.1016/j.ejrad.2011.10.029. [PubMed: 22119555].

23. de Ledinghen V, Vergniol J, Foucher J, Merrouche W, le Bail B. Noninvasive diagnosis of liver steatosis using controlled attenuation parameter (CAP) and transient elastography. Liver Int. 2012;32(6):911-8. doi: 10.1111/j.1478-3231.2012.02820.x. [PubMed: 22672642].

24. Lee JH, Kim D, Kim HJ, Lee CH, Yang JI, Kim W, et al. Hepatic steatosis index: a simple screening tool reflecting nonalcoholic fatty liver disease. Dig Liver Dis. 2010;42(7):503-8. doi:10.1016/j.dld.2009.08.002. [PubMed: 19766548].

25. McPherson S, Jonsson JR, Cowin GJ, O'Rourke P, Clouston AD, Volp A et al. Magnetic resonance imaging and spectroscopy accurately estimate the severity of steatosis provided the stage of fibrosis is considered.J Hepatol. 2009;51(2):389-97. doi: 10.1016/j.jhep.2009.04.012. [PubMed: 19505740]

26. Schwenzer NF, Springer F, Schraml C, Stefan N, Machann J, Schick F. Non-invasive assessment and quantification of liver steatosis by ultrasound, computed tomography and magnetic resonance. J Hepatol. 2009;51(3):433-45. doi: 10.1016/j.jhep.2009.05.023. [PubMed: 19604596]. 